\title{
COMETARY ION DISTRIBUTIONS NEAR THE PICKUP ENERGY OUTSIDE COMET HALLEY'S BOW SHOCK
}

\author{
T. I. Gombosi, ${ }^{*}$ M. Neugebauer, ${ }^{* *}$ A. D. Johnstone, ${ }^{* * *}$ \\ A. J. Coates*** and D. E. Huddleston*** \\ * Space Physics Research Laboratory, The University of Michigan, Ann Arbor, \\ MI 48109, U. S. A. \\ ** Jet Propulsion Laboratory, California Institute of Technology, 4800 Oak \\ Grove Drive, Pasadena, CA 91109, U.S. A. \\ *** Mullard Space Science Laboratory, University College London, Holmbury \\ St. Mary, Dorking, Surrey RH5 6NT, U.K.
}

\section{ABSTRACT}

This paper compares calculated and measured energy spectra of implanted $\mathrm{H}^{+}$and $\mathrm{O}^{+}$ions on the assumption that the pick-up geometry is quasi-parallel and about $1 \%$ of the waves generated by the cometary pickup process propagates backward (towards the comet). The model provides a good description of the implanted $\mathrm{O}^{+}$and $\mathrm{H}^{+}$energy distribution near the pickup energies. The thickness of the implanted ion velocity distribution shells was nearly constant between $2.50 \times 10^{6} \mathrm{~km}$ and $1.20 \times 10^{6} \mathrm{~km}$ (just outside the shock) along the inbound Giotto trajectory. The explanation is that the velocity diffusion coefficient and characteristic diffusion time vary approximately as $1 / \mathrm{r}$ and $\mathrm{r}$, respectively, therefore their product (which determines the velocity shell thickness) remains nearly constant.

\section{INTRODUCTION}

Instruments at comets Giacobini-Zinner and Halley /12,13,15,16/ detected large fluxes of energetic particles. A significant part of the observed energetic ion population was observed at energies considerably larger than the pickup energy indicating the presence of some kind of acceleration process acting on implanted ions. Velocity diffusion of lower energy implanted ions (near the pickup energy) has also been observed by the several instruments upstream of the comet Halley bow shock $13,21 \%$.

This paper compares observed implanted proton and oxygen distribution functions to the predictions of the generalized transport model $/ 9 \%$. The study is based on proton data from the HERS sensor of the Giotto Ion Mass Spectrometer (IMS) and on oxygen data obtained by the IIS sensor of the JPA instrument. The data cover the low energy superthermal particle population near the implanted ion peak $\left(\mathrm{E}_{\mathrm{H}}+<4 \mathrm{keV}\right.$ and $\left.\mathrm{E}_{\mathrm{O}}+<100 \mathrm{keV}\right)$ and do not extend to energies attained by significantly accelerated cometary ions.

\section{OBSERVATIONS}

The pickup proton data used in this study were obtained by the high-energy-range spectrometer (HERS) of the Giotto ion mass spectrometer (IMS). The IMS has been described by Balsiger et al. /1/. HERS was a magnetic mass spectrometer of rather unusual design which was capable of mapping 3-dimensional velocity distributions as a function of ion mass/charge for ions with energy/charge below $\sim 4 \mathrm{kV}$. Thus proton distributions could be studied without contamination or confusion by other ion species; when the spacecraft was upstream of the Halley bow shock, picked-up cometary protons could be distinguished from solar wind protons by their different locations in velocity space (i.e., by their very different velocity distributions). The method used to analyze the data reported here was similar to that described in $/ 20 \%$.

For the water group ions, we use data in the energy/charge range 2.5 to $55 \mathrm{kV}$ from the implanted ion sensor (IIS) of the Johnstone plasma analyzer (JPA) $/ 14,23 /$. This instrument was a time-of-flight mass spectrometer which measured the energy/charge and the velocity of individual ions. By measuring the output of five separate sensors as a function of the spacecraft spin phase, 3-dimensional observations of the ion distribution function were obtained. The "water group" data used in the present analysis correspond to ions with mass/charge in the range 6.5 to $20 \mathrm{amu} / \mathrm{q}$; upstream of the cometary bow shock the great majority of these ions were presumably $\mathrm{O}^{+}$ions. The method of data analysis is similar to that described by Coates et al. $/ 3 /$, taking into account the correction factors described by Coates et al. $12 \%$.

MODEL

Energetic particles in the cometary environment are most likely $\mathrm{O}^{+}$ions of cometary origin (cf. $/ 17 / /$ ). Before being ionized (by photoionization or charge exchange) these particles were escaping the comet with velocities significantly smaller than the solar wind speed. The freshly ionized cometary particles form a pickup ring distribution in the magnetized solar wind plasma within a fraction of a gyroperiod $/ 6 \%$. This rapid ring formation is followed by somewhat slower pitch-angle diffusion and much slower energy diffusion processes $16,22,24 /$. Only near the cometary bow shock does the pitch-angle diffusion due to pickup generated MHD turbulence become strong enough to result in nearly isotropic shell distributions (in the plasma frame) $13,21 /$ : further upstream, the implanted ion distribution functions are non-uniformly-filled and slightly broadened shells in velocity space. 
The present transport model is based on a series of recent papers discussing implanted ion acceleration processes in the upstream cometary region $/ 8,9 \%$. The spatial and velocity diffusion coefficients were obtained by using the quasilinear approximation and were discussed in datail in earlier papers $/ 9,10 \%$. In the present calculation Equation (1) was solved for implanted cometary $\mathrm{H}^{+}$and $\mathrm{O}^{+}$ions. The $\overrightarrow{\mathrm{u}}$ and $\mathrm{B}$ vectors were assumed to be known functions of the spatial coordinate, $\vec{r}$. These functions were taken from a 3D cometary MHD model $/ 5 /$. In our particular case the upstream flow velocity was $u=300 \mathrm{~km} / \mathrm{s}$, and the particle density was $n_{S w}=6 \mathrm{~cm}^{-3}$. Equation (2) was numerically solved using an alternating direction fully implicit numerical scheme with second order accuracy. The same numerical technique was applied in an earlier paper $/ 9 /$ and some important details are discussed there.

\section{RESULTS AND DISCUSSION}

Implanted ion distributions were calculated using the model described in the previous section. The model solved the transport equation along flow lines (which in this particular model are identical to magnetic field lines). For each flow line the calculation started at $2.5 \times 10^{6} \mathrm{~km}$ upstream from the point where the flow line intersected the Giotto trajectory, and it extended to a distance of $1.5 \times 10^{6} \mathrm{~km}$ downstream from the intersection point. The $4 \times 10^{6} \mathrm{~km}$ distance (along the curved flow line) was divided into 200 spatial grid points resulting in a $2 \times 10^{4} \mathrm{~km}$ spatial step size. The velocity interval extended from 0 to $3500 \mathrm{~km} / \mathrm{s}$ (in the plasma frame) with a $25 \mathrm{~km} / \mathrm{s}$ step size. There were free escape boundaries at both ends of the flow line, and there was no flux through $v=0$ and $v=3500 \mathrm{~km} / \mathrm{s}$ (this velocity was high enough so that only an insignificant number of particles was accelerated to this value).

The model predictions were compared to observations made by the IMS HERS and JPA IIS instruments. Fig. 1 shows a comparison of the measured and calculated velocity space shell thicknesses along the inbound portion of the Giotto trajectory. In Fig. 1, solid and dotted lines represent the calculated velocity shell thickness of implanted $\mathrm{O}^{+}$and $\mathrm{H}^{+}$ions, respectively. Filled circles correspond to measured $\mathrm{H}^{+}$velocity shell thicknesses, while triangles represent observed shell thicknesses of implanted $\mathrm{O}^{+}$spectra.

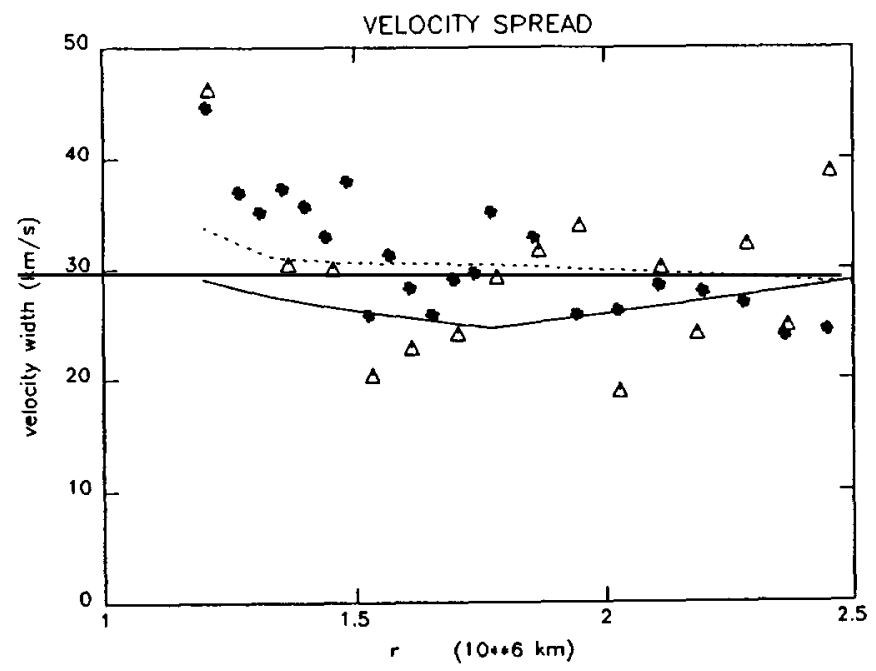

Fig. 1. Comparison of calculated and observed shell thicknesses of implanted ions. Measured values of $\mathrm{H}^{+}$and $\mathrm{O}^{+}$ are represented by filled circles and triangles, respectively. The calculated curves are represented by dotted $\left(\mathrm{H}^{+}\right)$and solid lines $\left(\mathrm{O}^{+}\right)$. Distance is measured in units of $10^{6} \mathrm{~km}$, while the number density values are given in $\mathrm{cm}^{-3}$.

Inspection of Fig. 1 reveals that between about $2.5 \times 10^{6} \mathrm{~km}$ and $1.2 \times 10^{6} \mathrm{~km}$ the shell thicknesses of both water group ions and implanted protons are in reasonably good agreement with the model predictions. Just outside the shock (at a cometocentric distance of about $1.2 \times 10^{6} \mathrm{~km}$ ) both velocity shell thicknesses suddenly increase. This increase might be an indication that the model loses validity near the shock, where microphysical processes might result in particle acceleration mechanisms not accounted for in the present model. While it would be dangerous to base far reaching conclusions on one data point, it is significant that the increase at this point is part of the overall rise associated with the bow shock /4/. Fig. 1 also shows that over the whole region examined, the calculated hydrogen velocity shell thickness is quite close to the observed values, even though the data exhibit a somewhat steeper increase than the model prediction.

In spite of the good agreement between model prediction and observations this result has to be viewed with some scepticism. First of all, in this region water group ions pitch-angle scatter more than the protons (because there is much more wave power near the heavy ion cyclotron frequency) $/ 18 \%$. The water group ions form a more or less isotropic shell distribution in this region 3,4 , while the implanted proton distribution remains quite anisotropic almost up to the shock crossing /19/). Even though the present model does not assume a perfectly isotropic velocity space shell distribution, it does not allow for anisotropies which are large compared to the isotropic term. The model also assumes that the magnetic field vector is parallel to the flow velocity: this condition was not satisfied all the time during the part of the Giotto trajectory shown in Fig. 1. Finally, it should be noted that the measured velocity widths shown in Fig. 1 are upper limits to broadening due to wave-particle interaction, because the observational data include instrumental broadening (which might be different for protons and water group ions), as well as the effects of solar wind and magnetic field variations. 
An interesting feature of the calculated velocity shell thicknesses is their very small variation between about 2.5 and 1.2 million $\mathrm{km}$ cometocentric distances along the Giotto trajectory. As can be seen in Fig. $1\langle\Delta v\rangle_{\mathrm{o}^{+}}$varies only about $10 \%$ over this range, while $\langle\Delta \mathrm{v}\rangle_{\mathrm{H}^{+}}$increases slowly until right before the shock. The near constancy of $\langle\Delta v\rangle$ is especially interesting, because the velocity diffusion coefficient increases about a factor of 10 over this interval.

The variation (or lack of variation) of implanted proton and water group velocity shell thicknesses with cometocentric distance is closely related to the variation of ion production rates along individual plasma flow lines. Upstream of the comet terminator plane local production dominates, which means that due to the rapid growth of local production rate the bulk of the implanted particle population was produced very recently, within approximately $\tau=u / L$, where $L$ is the characteristic length scale of ion implantation along the flow line. This means that the freshly implanted ion velocity distribution spreads approximately $\langle\Delta v\rangle \approx(D \tau)^{1 / 2}$ before being overtaken by the increasing number of continuously produced particles. As the plasma volume element is transported downstream of the comet terminator plane the local production rate decreases and the time available for velocity diffusion becomes $\tau \approx u / x-x_{c} l$, where $x_{c}$ is the location of the cometary nucleus. Along the Giotto trajectory this means that $\tau \approx 2.9 \mathrm{u} / \mathrm{r}$, where $\mathrm{r}$ is the cometocentric distance (the factor of 2.9 is $\left.1 / \cos 70^{\circ}\right)$. Near the pickup velocity $(v \approx 300 \mathrm{~km} / \mathrm{s})$ the implanted proton diffusion coefficient increases nearly linearly as one approaches the comet. The water group ion diffusion coefficient is also more or less linear, but it changes slope at around $1.8 \times 10^{6} \mathrm{~km}$. This means that the velocity spread is more or less constant along the incoming Giotto trajectory between $2.5 \times 10^{6} \mathrm{~km}$ and $1.2 \times 10^{6} \mathrm{~km}$, because $\langle\Delta \mathrm{v}\rangle \approx(\mathrm{D} \tau)^{1 / 2} \approx$ constant.

The agreement between the model and observations is quite remarkable considering the simplifying assumptions (parallel $\vec{u}(\vec{r})$ and $B(\vec{r})$ vectors, quasilinear diffusion coefficients, etc.) and experimental uncertainties. It should also be emphasized that a single set of physical parameters were used to describe a large number of implanted ion spectra. The adopted solar wind parameters were quite close to the observed values (although $u_{s w}=300 \mathrm{~km} / \mathrm{s}$ is probably on the low side), and the gas production rate was observed to be $Q_{n}=6.9 \times 10^{29} \mathrm{~s}^{-1}$ during the Giotto encounter (however, the appropriate value of $Q_{n}$ may be somewhat higher, because the neutral particle fluxes at millions of kilometers were emitted a few days earlier, when the comet was more active $/ 11 /$ ). The adopted ionization scale length values are very close to the ones estimated by the Giotto IMS and JPA teams /20,4/ and the observed spectral index of pickup generated magnetic field fluctuations $\eta /$ is also consistent with our value of 2 . The choice of $\alpha_{\text {com }}=0.01$ seems to be arbitrary, but is supported by recent plasma kinetic simulations $/ 18 /$.

\section{SUMMARY}

The results of model calculations describing transport and energization of cometary pickup ions were compared to observations made by the Giotto spacecraft. The model takes into account adiabatic compression, energy diffusion, field aligned spatial diffusion and mass addition along flow lines of decelerating plasma flow lines. The spatial distributions of plasma bulk velocity and the magnetic field were taken from a 3-dimensional MHD calculation, and it was assumed that the plasma flow velocity and magnetic field were parallel at all spatial locations. The model used the quasilinear approximation of the velocity and spatial diffusion coefficients. The generalized transport equation for the velocity-space solid-angle-averaged distribution function was solved for individual MHD flow lines. The implanted ion spectra at the intersection points of the flow line with Giotto's trajectory were compared with spectra observed at that particular spatial location. The main conclusions of this comparison are:

1. Assuming that about $1 \%$ of the waves generated by the cometary pickup process propagates backward (towards the comet) the theoretical model provides a good description of the implanted $\mathrm{O}^{+}$energy distribution near the pickup energy. For implanted protons there is some disagreement between observations and model prediction outside of about $1.4 \times 10^{6} \mathrm{~km}$ due to a combination of effects including anisotropic velocity space shell filling.

2. The calculated thickness of the implanted ion velocity distribution shells was only slowly increasing between $2: 50 \times 10^{6} \mathrm{~km}$ and $1.20 \times 10^{6} \mathrm{~km}$ (just outside the shock) along the inbound Giotto trajectory, which implies that the velocity diffusion coefficient was nearly constant in an extended region outside the cometary bow shock. The explanation is that the velocity diffusion coefficient and characteristic diffusion time vary approximately as $1 / \mathrm{r}$ and $\mathrm{r}$, respectively, therefore their product (which determines the velocity shell thickness) remains nearly constant.

\section{ACKNOWLEDGEMENTS}

The authors are indebted to Drs. J.G. Luhmann, J.A. Fedder, H.U. Schmidt, and R. Wegmann for providing 3D MHD flow field models. This work was supported by NASA grants NAGW-1366 and NAGW-2162 at the University of Michigan. Part of the research described in this paper was carried out by the Jet Propulsion Laboratory of the Califomia Institute of Technology under contract with the US. National Aeronautics and Space Administration. The work at the Mullard Space Science Laboratory was supported by the Science and Engineering Research Council of the United Kingdom, and by a research fellowship for AJC from the Royal Society of the United Kingdom. Acknowledgement is also made to the National Center for Atmospheric Research sponsored by NSF, for the computing time used in this research.

\section{REFERENCES}

1. Balsiger, H., et al., The Giotto Ion-mass spectrometer, J. Phys. E., 20,759, 1987.

2. Coates, A.J., et al., Correction to "Velocity space diffusion of pickup ions from the water group at comet Halley", J. Geophys. Res., 95, 4343, 1990a.

3. Coates, A.J., et al., Velocity-space diffusion of pickup ions from water group at Comet Halley, J. Geophys. Res., 94, 9983, 1989.

4. Coates, A.J., et al., Bulk properties and velocity distributions of water group ions at comet Halley: Giotto 
measurements, J. Geophys. Res., 95, 1990b.

5. Fedder, J.A., et al., EOS, Trans. $A G U, 67,17,1986$.

6. Gaffey, J., J.D., et al., Time scales for formation and spreading of velocity shells of pickup ions in the solar wind, J. Geophys. Res., 93, 5470, 1988.

7. Glassmeier, K.-H., et al., Spectral characteristics of low-frequency plasma turbulence upstream of comet P/Halley, J. Geophys. Res., 94, 37, 1989.

8. Gombosi, T.I., Preshock region acceleration of implanted cometary $\mathrm{H}^{+}$and $\mathrm{O}^{+}, J$. Geophys. Res., 93, 35, 1988.

9. Gombosi, T.I., et al., Combined first- and second-order Fermi acceleration in cometary environments, $J$. Geophys. Res., 94, 15011, 1989.

10. Gombosi, T.I., et al., Comparison of observed and calculated implanted ion distributions outside comet Halley's bow shock, J. Geophys. Res., 1990.

11. Huddleston, D.E., et al., Determination of comet Halley gas emission characteristics from mass loading of the solar wind, J. Geophys. Res., 95, 21, 1990.

12. Hynds, R.J., et al., Observations of energetic ions from comet Giacobini-Zinner, Science, 232, 361, 1986.

13. Ipavich, F.M., et al., Comet Giacobini-Zinner: In situ observations of energetic heavy ions, Science, 232,366 , 1986.

14. Johnstone, A.D., et al., The Giotto three-dimensional positive ion analyzer, J. Phys. E, 20, 795, 1987.

15. Kecskeméty, K., et al., Pickup ions in the unshocked solar wind at comet Halley, J. Geophys. Res., 94, 185, 1989.

16. McKenna-Lawlor, S., et al., In situ energetic particle observations at comet Halley recorded by instrumentation aboard the Gionto and Vega 1 missions, Annales Geophysicae, 7, 121, 1989.

17. Mendis, D.A., et al., The physics of comets, Fund. Cosmic Phys., 10, 1, 1985.

18 Miller, R.H., et al., The directional dependence of magnetic fluctuations generated by cometary ion pickup, $J$. Geophys. Res., submitted, 1990.

19. Neugebauer, M., et al., Comparison of picked-up protons and water group ions upstream of comet Halley's bow shock, J. Geophys. Res., 1990.

20. Neugebauer, M., et al., The density of cometary protons upstream of comet Halley's bow shock, J.Geophys. Res., 94, 1261, 1989a.

21. Neugebauer, M., et al., The velocity distribution of cometary protons picked up by the solar wind, J. Geophys. Res., 94, 5227, 1989b.

22. Sagdeev, R.Z., et al., MHD turbulence in the solar wind - comet interaction region, Geophys. Res. Lett., 13, 85,1986

23. Wilken, B., et al., The Giotto implanted ion spectrometer (IIS): physics and technique of detection, J. Phys. E., $20,778,1987$.

24. Winske, D., et al., Coupling of newborn ions to the solar wind by electromagnetic instabilities and their interaction with the bow shock, J. Geophys. Res., 90, 2713, 1985. 